Commun. Korean Math. Soc. 29 (2014), No. 1, pp. 213-218

http://dx.doi.org/10.4134/CKMS.2014.29.1.213

\title{
ON THE SIMPLICITY OF THE CODED PATH OF THE CODE $(i)$
}

\author{
Dal Young JeOng And Jeong Suk Son
}

\begin{abstract}
J. Malkevitch defined the coded path in $r$-valent polytopal graphs of uniform face structure and showed many interesting properties of the coded paths. In this paper, we study the simplicity of coded paths in an $m$-valent planar multigraph which is not a polytopal graph.
\end{abstract}

\section{Introduction}

In this section, we introduce some definitions and theorems briefly.

A graph $G$ is an ordered triple $\left(V(G), E(G), \psi_{G}\right)$ consisting of a nonempty set $V(G)$ of vertices, a set $E(G)$ of edges, and an incidence function $\psi_{G}$ that associates with each edge of $G$ an unordered pair of (not necessarily distinct) elements of $V(G)$. If $e$ is an edge and $u$ and $v$ are vertices such that $\psi_{G}(e)=u v$, then $e$ is said to join two vertices $u$ and $v$; the vertices $u$ and $v$ are called the endpoints (or endvertices) of the edge $e$. The endpoints of an edge are said to be incident to an edge and two vertices which are incident with the same edge, are said to be adjacent.

Two or more edges that join the same pair of distinct vertices are called parallel edges (or multiple edges). An edge joining a vertex to itself is called a loop. A graph with no loops or no parallel edges is called a simple graph. A graph which is not simple is said to be a multigraph.

The number of edges at the vertex $v$ is called the valence of $v$ (or the degree of $v$ ) and is denoted by $d(v)$. If every vertex of a graph $G$ has the same valence $r$, then $G$ is called an $r$-valent (or $r$-regular) graph.

A graph $G$ is called planar if it can be drawn in the plane so that the edges of the graph intersect only at vertices. When a connected planar graph is drawn in the plane, the regions bounded by edges of the graph which do not contain neither vertices nor edges in their interiors are called faces. There will always be precisely one face which is unbounded in a planar graph and this will be called the infinite face. The edges bounding a face are called its sides.

Received October 11, 2013.

2010 Mathematics Subject Classification. 05C10, 05C38, 52C30.

Key words and phrases. coded path, universally simple, multi 2-gonal graphs. 
Suppose $G$ is a planar connected graph. A face $F$ with $k$ sides will be called a $k$-gon. If the number of sides of $F$ is a multiple of $k$, it will be called a multi $k$-gon. If all the faces of a graph $G$ are $k$-gons (or multi $k$-gons), then $G$ will be called a $k$-gonal (or multi $k$-gonal, respectively) graph.

A bipartite graph is one whose vertex set can be partitioned into two disjoint subsets $X$ and $Y$, so that each edge has one endpoint in $X$ and the other endpoint in $Y$.

For other things which are not mentioned here, we refer to Bondy \& Murty [1] or G. Chartrand [3].

We state a few theorems for further reading without proof.

Theorem 1.1. A graph is bipartite if and only if it contains no odd cycle.

Theorem 1.2. A graph $G$ is bipartite if and only if $G$ is 2-colorable.

J. Malkevitch defined a "coded path" in an $r$-valent polytopal graph [2] . Similarly, we define a "coded trail" in an $r$-valent multigraph.

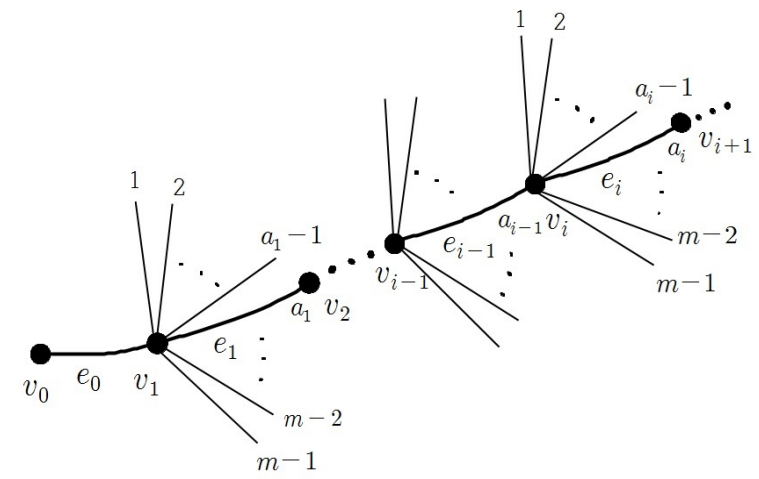

Figure 1. A coded path $\left(a_{1}, a_{2}, \ldots, a_{n}\right)$

Let $G$ be a connected planar $r$-valent $(r \geq 2)$ multigraph which has no loops. We consider an ordered $n$-tuple $\left(a_{1}, \ldots, a_{n}\right)$ of integers such that $1 \leq$ $a_{i} \leq r-1, i=1,2, \ldots, n$. We call such a sequence $a$ code of length $n$.

Let $e_{0}$ be any edge of $G$ with endpoints $v_{0}$ and $v_{1}$. The coded trail in the direction from $v_{0}$ to $v_{1}$ determined by the code $C$ is an alternating sequence of vertices and edges

$$
v_{0}, e_{0}, v_{1}, e_{1}, v_{2}, \ldots, v_{k-1}, e_{k}, v_{k}, \ldots
$$

where each edge is chosen as follows (Figure 1): At the vertex $v_{i}, i \geq 1$, there are $r-1$ edges incident to the vertex $v_{i}$ except the edge $e_{i-1}$. Those edges can be numbered from left to right starting with 1 based on the direction from $v_{i-1}$ to $v_{i}$. Then we choose the $a_{j}$-th $($ where $j \equiv i(\bmod n))$ edge as the next edge 
$e_{i}$ (if $j \equiv 0$, choose $a_{n}$-th edge) and let $v_{i+1}$ be the other endpoint of the edge $e_{i}$ (Figure 1).

The trail terminates with the edge $e_{i}$ if the followings are satisfied:

(a) There is some $i$ such that $i \equiv n-1(\bmod n)$ and $e_{i+1}=e_{0}$;

(b) the choice for $e_{i+2}$ would be $e_{1}$.

A trail determined by the code $C$ is said to be a $C$-trail. If all the vertices in a $C$-trail are distinct, it is called a $C$-path. If $C$-path ends up with a cycle, we say that the $C$-path is simple with respect to the directed edge $e_{0}=v_{0} v_{1}$.

Figure 2 shows the $(1,2)$-path associated with the edge $e_{0}=v_{0} v_{1}$ in a 4 valent graph.

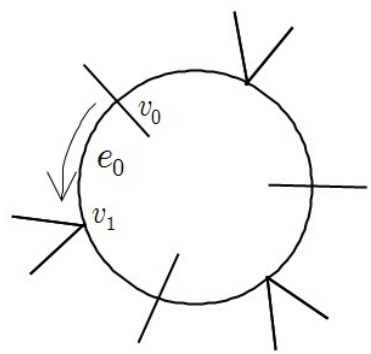

Figure 2. A coded path $(1,2)$

If, for every edge $e=v_{0} v_{1}$ of $G$, the $C$-paths associated with the directed edges $v_{0} v_{1}$ and $v_{1} v_{0}$ are simple, then $C$ is said to be universally simple with respect to $G$. Note that the code $(1)$ and the code $(r-1)$ of an $r$-valent graph $G$ are universally simple with respect to $G$ if $G$ is 2-connected. Actually, each of the simple $C$-paths associated with the directed edge $v_{0} v_{1}$ uniquely determines a face of the graph $G$.

\section{Main results}

Now, we study the simplicity of a $C$-trail where $C=(i), 1 \leq i \leq m-1$ in an $m$-valent graph.

Theorem 2.1. For $m \geq 2$, let $G$ be an $m$-valent planar multi 2-gonal multigraph. Then the code $(i)$ is universally simple with respect to $G$.

Proof. Since $G$ has only even cycles, it is bipartite. Since $m \geq 2, G$ is 2 connected and so two codes $(1)$ and $(m-1)$ are universally simple. Therefore we only consider the code $(i)$ for $2 \leq i \leq m-2$.

Suppose that $(i)$-trail does not end up with a cycle for some $i \in\{2, \ldots, m-$ $2\}$. Then there exists a walk that is made by $(i)$-trail associated with a directed edge $e_{0}=v_{0} v_{1}$, which has at least one crossing vertex as shown in Figure 3. 


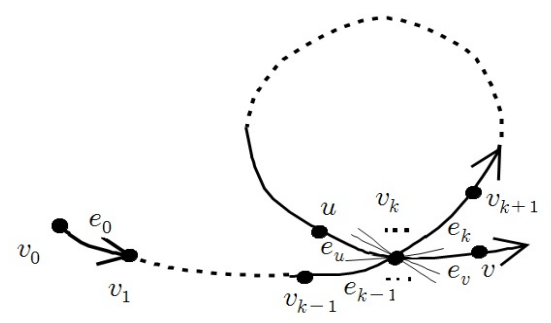

Figure 3. A walk that is made by $(i)$-trail

Let $v_{k}$ be a crossing vertex and let the edge join the vertices $v_{k-1}$ and $v_{k}$ be $e_{k-1}$ (Figure 3). Now, label $m-1$ edges incident to the vertex $v_{k}$ except $e_{k-1}$ from left to right with numbers $1,2, \ldots, m-1$. Let $j$ be the label of the edge which the $(i)$-trail chooses when it visits the vertex $v_{k}$ again.

First, we consider the case where $1 \leq j \leq i-1$. Then an $(i)$-trail has a subtrail as follows (Figure 4):

$$
\cdots v_{k-1} e_{k-1} v_{k} e_{k} v_{k+1} \cdots u e_{u} v_{k} e_{v} v \cdots
$$
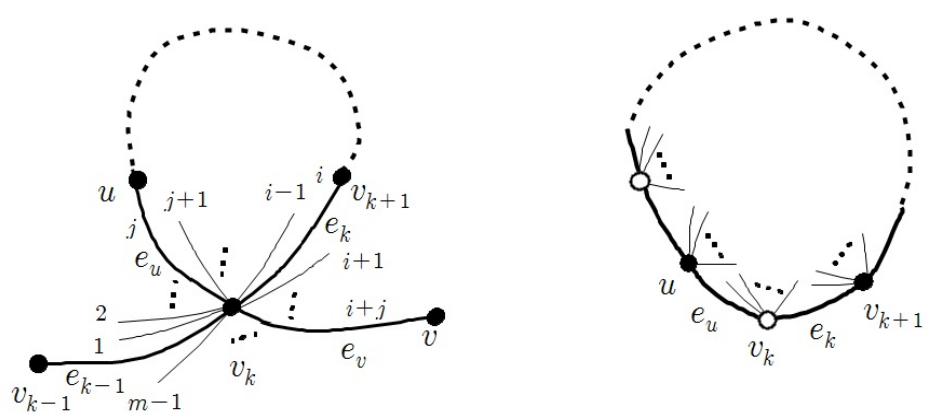

Figure 4 . The case where $1 \leq j \leq i-1$

Let $C^{\prime}=v_{k} e_{k} v_{k+1} \cdots u e_{u} v_{k}$, then it is a cycle. For our proof, delete all the vertices outside of $C^{\prime}$. Then we have a subgraph $G^{\prime}$ whose vertices are either on or inside the cycle $C^{\prime}$ and each vertex on the cycle is of degree $i+1$ except the vertex $v_{k}$ whose degree is $i-j+1$ (Figure 4). Since the graph $G$ is bipartite, so is $G^{\prime}$. Hence the vertices of $G^{\prime}$ can be colored by two colors, say white and black. We may assume that the vertex $v_{k}$ is a white vertex and the length of the cycle $C^{\prime}$ is $2 p$ for some positive integer $p$ without loss of generality. Let the 
numbers of the black vertices and the white vertices inside the cycle be $n_{b}$ and $n_{w}$, respectively. Then the sum of degrees of the black vertices in $G^{\prime}$ is

$$
(i+1) \times p+m \times n_{b},
$$

while the sum of degrees of the white vertices is

$$
(i+1) \times(p-1)+(i-j+1)+m \times n_{w} .
$$

Since $G^{\prime}$ is bipartite, each edge joins a black vertex to a white vertex without making any intersection. So the sum of the degrees of the black vertices must be equal to the sum of the degrees of the white vertices:

$$
(i+1) \times p+m \times n_{b}=(i+1) \times(p-1)+(i-j+1)+m \times n_{w},
$$

which is simplified into

$$
m \times\left(n_{w}-n_{b}\right)=j .
$$

However, it is impossible because the left hand side is a multiple of $m$ while the right hand side is not a multiple of $m(1 \leq j \leq i-1<m)$.

Now, let's consider the case where $i+1 \leq j \leq m-1$. By applying a similar argument for the case $1 \leq j \leq i$, we can get a cycle $C^{\prime}$ of length $2 p$ for some positive integer $p$. We delete the vertices outside $C^{\prime}$ to obtain a graph $G^{\prime}$. Then the degree of each vertex except $v_{k}$ is $m-i+1$ while the degree of the vertex $v_{k}$ is $j-i+1$ in $G^{\prime}$. By applying the same argument for the case $1 \leq j \leq i$, we obtain the following equality.

$$
(m-i+1) \times p+m \times n_{b}=(m-i+1) \times(p-1)+(j-i+1)+m \times n_{w},
$$

which is simplified into

$$
m \times\left(n_{w}-n_{b}\right)=m-j .
$$

Clearly, this is impossible because the left hand side is a multiple of $m$ while the right hand side is not a multiple of $m$.

Thus, an $(i)$-trail is simple with respect to the directed edge $e_{0}=v_{0} v_{1}$.

Similarly, we can show that an $(i)$-trail associated with the directed edge $e_{0}=v_{1} v_{0}$ ends up with a cycle. Thus, the code $(i)$ is universally simple with respect to $G$.

The following theorem provides more information on vertices inside the cycle formed by the $(i)$-path.

Theorem 2.2. For $m \geq 2$, let $G$ be an m-valent planar bipartite graph with a bipartition $(X, Y)$ and let $C$ be a cycle in $G$. If each vertex on the cycle $C$ is incident to the same number of edges inside the cycle $C$, then the number of the vertices of $X$ which are inside the cycle is equal to the number of vertices of $Y$ which are inside the cycle.

Proof. Since $G$ is a bipartite graph, every cycle in $G$ is of even length and all the vertices are colored properly with black and white. Let $C$ be a cycle of length $2 p$ for some positive integer $p$ and let $k$ be the number of edges inside 
the cycle $C$ incident to each vertex on the cycle $C$. Clearly, the number of black vertices and the number of white vertices on the cycle $C$ are equal.

Delete all the vertices outside the cycle $C$. Let $n_{b}$ and $n_{w}$ be the number of the black vertices and the number of the white vertices inside the cycle, respectively. Since $G$ is bipartite, each edge must join a black vertex and a white vertex without making an intersection. So the number of edges incident to black vertices and the number of edges incident to white vertices must be equal. This means that the degree sum of the black vertices and the degree sum of the white vertices are equal. Hence we have the following equation

$$
(k+2) \times p+m \times n_{b}=(k+2) \times p+m \times n_{w},
$$

which yields

$$
n_{b}=n_{w}
$$

Therefore, the number of the vertices of $X$ which are inside the cycle is equal to the number of vertices of $Y$ which are inside the cycle.

Acknowledgements. We would like to thank referee for his/her detailed and helpful comments.

\section{References}

[1] J. A. Bondy and U. S. R. Murty, Graph Theory with Applications, Elsevier Publishing Co., 1977.

[2] J. Malkevith, Properties of planar graphs with uniform vertex and face structure, Memoirs of the A.M.S., American Mathematical Society, 1970.

[3] G. Chartrand and L. Lensniak, Graphs and Digraphs, Wadsworth and Books, California, 1979.

Dal Young Jeong

Department of Mathematics

SOONGSIL UNIVERSITY

SEOUl 156-743, Korea

E-mail address: dyjeong@ssu.ac.kr

JEONG SUK SON

Department of Mathematics

SOONGSIL UNIVERSITY

Seoul 156-743, Korea

E-mail address: helper0608@ssu.ac.kr 